\title{
Fuzzy Extension of Coupled $\Gamma$-semiring: Some Properties
}

\section{Clement Boateng Ampadu}

31 Carrolton Road, Boston, MA 02132-6303, USA; e-mail: drampadu@ hotmail.com

\begin{abstract}
The concept of coupled $\Gamma$-semiring first appeared in [1]. In the present paper assuming $M$ is a $\Gamma$-semiring, we introduce concepts of anti-fuzzy prime ideal, anti-fuzzy semi prime ideal, and anti-fuzzy ideal extension, respectively, of $M \times M$. Some properties associated with these new concepts are obtained. The work in this paper takes inspiration from [2].
\end{abstract}

\section{Introduction and Preliminaries}

Definition 1.1. [2] A fuzzy subset $\mu$ of a $\Gamma$-semiring $M$ is called an anti-fuzzy left ideal of $M$, if the following hold:

(a) $\mu(x+y) \leq \max \{\mu(x), \mu(y)\}$

(b) $\mu(x \alpha y) \leq \mu(y)$

for all $x, y \in M, \alpha \in \Gamma$.

Remark 1.2. [2] If we replace (b) in the above definition with $\mu(x \alpha y) \leq \mu(x)$, then we say the fuzzy subset $\mu$ of the $\Gamma$-semiring $M$ is an anti-fuzzy right ideal of $M$.

Remark 1.3. [2] If $\mu$ is an anti-fuzzy left (right) ideal of a $\Gamma$-semiring $M$, then $\mu(0) \leq \mu(x)$ for all $x \in M$.

Received: January 2, 2020; Accepted: March 30, 2020

2010 Mathematics Subject Classification: 16Y60, 16Y99, $03 E 72$.

Keywords and phrases: coupled, $\Gamma$-semiring, anti-fuzzy, ideal, prime, semi-prime, extension.

Copyright (C) 2020 Clement Boateng Ampadu. This is an open access article distributed under the Creative Commons Attribution License, which permits unrestricted use, distribution, and reproduction in any medium, provided the original work is properly cited. 
Definition 1.4. [2] A fuzzy subset $\mu$ of a $\Gamma$-semiring $M$ is called an anti fuzzy ideal of $M$, if $\mu$ is both an anti fuzzy left and anti-fuzzy right ideal of $M$.

Definition 1.5. [2] A subset $A$ of a $\Gamma$-semiring $M$ is a left (right) ideal of $M$, if $A$ is an additive semigroup of $M$, and the set

$$
M \Gamma A=\{x \alpha y \mid x \in M, \alpha \in \Gamma, y \in A\} \quad(A \Gamma M)
$$

is contained in $A$. If $A$ is both a left and right ideal of $M$, then $A$ is an ideal of $M$.

Definition 1.6. [2] Let $M$ be a $\Gamma$-semiring, and $\mu$ be an anti-fuzzy ideal of $M$. Then $\mu$ is an anti-fuzzy prime ideal of $M$, if $\mu(x) \leq \mu(x \alpha x)$ for all $x \in M$, and $\alpha \in \Gamma$.

Definition 1.7. [2] Let $M$ be a $\Gamma$-semiring, and $\mu$ be a fuzzy subset of $M$. The fuzzy subset $\langle x, \mu\rangle: M \mapsto[0,1]$ defined by

$$
\langle x, \mu\rangle(y)=\sup _{\alpha \in \Gamma} \mu(x \alpha y)
$$

for all $y \in M$ is called an extension of $\mu$ by $x$.

Definition 1.8. [2] Let $M$ be a $\Gamma$-semiring, and $\mu$ be a fuzzy subset of $M$. Then $\mu$ is called an anti fuzzy prime ideal of $M$ if

$$
\mu(x \alpha y)=\min \{\mu(x), \mu(y)\}
$$

for all $x, y \in M$, and $\alpha \in \Gamma$.

Definition 1.9. [2] Let $M$ be a $\Gamma$-semiring, and $\mu$ be an anti fuzzy ideal of $M$. For any $t \in[0,1]$, we define $\mu_{t}$ by the set

$$
\{x \in M \mid \mu(x) \leq t\}
$$

and call $\mu_{t}$ an anti level subset.

Definition 1.10. [2] Let $M$ be a $\Gamma$-semiring, $A \subseteq M$, and $x \in M$. We define $\langle x, A\rangle$ by

$$
\{y \in M \mid x \alpha y \in A \text {, for all } \alpha \in \Gamma\} .
$$

Definition 1.11. [2] Let $M$ be a $\Gamma$-semiring, and $\mu$ be an anti-fuzzy ideal of $M$. We say $\mu$ is an anti fuzzy semi prime ideal if 


$$
\mu(x) \leq \mu(x \alpha x)
$$

for all $x \in M$.

Definition 1.12. [2] Let $M$ be a $\Gamma$-semiring. Then the fuzzy ideal $\mu$ of $M$ is called an anti fuzzy $k$-ideal of $M$ if

$$
\mu(x) \leq \max \{\mu(x+y), \mu(y)\}
$$

for all $x, y \in M$.

\section{Main Results}

Definition 2.1. Let $M$ be a $\Gamma$-semiring. A fuzzy subset $\mu$ of $M \times M$ is called an antifuzzy left ideal of $M \times M$, if the following hold:

(a) $\mu(x+y, m+v) \leq \max \{\mu(x, m), \mu(y, v)\}$

(b) $\mu(x \alpha y, m \alpha v) \leq \mu(y, v)$

for all $(x, m),(y, v) \in M \times M, \alpha \in \Gamma$.

Remark 2.2. If we replace (b) in the above definition with $\mu(x \alpha y, m \alpha y) \leq \mu(x, m)$, then we say the fuzzy subset $\mu$ of $M \times M$ is an anti-fuzzy right ideal of $M \times M$.

Remark 2.3. If $\mu$ is an anti-fuzzy left (right) ideal of $M \times M$, then $\mu(0,0) \leq$ $\mu(x, m)$ for all $(x, m) \in M \times M$.

Definition 2.4. A fuzzy subset $\mu$ of $M \times M$ is called an anti fuzzy ideal of $M \times M$, if $\mu$ is both an anti fuzzy left and anti-fuzzy right ideal of $M \times M$.

Definition 2.5. Let $M$ be a $\Gamma$-semiring, and define $M^{*}:=M \times M$. A subset $A$ of $M^{*}$ is called a left (right) ideal of $M^{*}$, if $A$ is an additive semigroup of $M^{*}$, and the set

$$
M^{*} \Gamma A=\left\{(x \alpha y, m \alpha v) \mid(x, m) \in M^{*}, \alpha \in \Gamma,(y, v) \in A\right\} \quad\left(A \Gamma M^{*}\right)
$$

is contained in $A$. If $A$ is both a left and right ideal of $M^{*}$, then $A$ is an ideal of $M^{*}$.

Theorem 2.6. Let $M$ be a $\Gamma$-semiring, and define $M^{*}:=M \times M$. Suppose $A$ is a nonempty subset of $M^{*}$, and define fuzzy subset $\mu$ in $M^{*}$ by 


$$
\mu(x, m)= \begin{cases}0 & \text { if }(x, m) \in A \\ 1 & \text { if }(x, m) \notin A .\end{cases}
$$

Then $\mu$ is an anti-fuzzy ideal of $M^{*}$ if and only if $A$ is an ideal of $M^{*}$.

Proof. Suppose $\mu$ is an anti-fuzzy ideal of $M^{*}$. Let $(x, m),(y, v) \in A$. It follows that $\mu(x, m)=\mu(y, m)=0$. Now observe that

$$
\mu(x+y, m+v) \leq \max \{\mu(x, m), \mu(y, v)\}=0 .
$$

Hence, $(x+y, z+m) \in A$. Now let $(x, m),(y, v) \in A$ and $\alpha \in \Gamma$, and observe that

$$
\mu(x \alpha y, m \alpha v) \leq \min \{\mu(x, m), \mu(y, v)\}=0 .
$$

Hence, $(x \alpha y, m \alpha v) \in A$. It now follows that $A$ is an ideal of $M^{*}$. For the converse, let $(x, m),(y, v) \in A, \alpha \in \Gamma$, and $A$ an ideal of $M^{*}$. We consider the following cases.

Case I. $(x, m) \in A$ and $(y, v) \in A$

In this case we know $\mu(x, m)=0$ and $\mu(y, v)=0$. Also since $A$ is an ideal of $M^{*}$, we know $(x+y, z+m) \in A$ and $(x \alpha y, m \alpha v) \in A$, thus, $\mu(x+y, m+v)=0$, and $\mu(x \alpha y, m \alpha v)=0$. Since $\max \{\mu(x, m), \mu(y, v)\}=0$, and, $\min \{\mu(x, m), \mu(y, v)\}=0$, we have, $0=\mu(x+y, m+v)=\max \{\mu(x, m), \mu(y, v)\}=0$, and $0=\mu(x \alpha y, m \alpha v)=$ $\min \{\mu(x, m), \mu(y, v)\}=0$, hence the conclusion.

Case II. $(x, m) \notin A$ and $(y, v) \notin A$

In this case we know $\mu(x, m)=1$ and $\mu(y, v)=1$. Also since $A$ is an ideal of $M^{*}$, we know $(x+y, z+m) \in A$ and $(x \alpha y, m \alpha v) \in A$, thus, $\mu(x+y, m+v)=0$, and $\mu(x \alpha y, m \alpha v)=0$. Since $\max \{\mu(x, m), \mu(y, v)\}=1$, and, $\min \{\mu(x, m), \mu(y, v)\}=1$, we have, $0=\mu(x+y, m+v)<\max \{\mu(x, m), \mu(y, v)\}=1$, and

$$
0=\mu(x \alpha y, m \alpha v)<\min \{\mu(x, m), \mu(y, v)\}=1
$$

hence the conclusion.

Case III. $(x, m) \in A$ and $(y, v) \notin A$ 
In this case we know $\mu(x, m)=0$ and $\mu(y, v)=1$. Also since $A$ is an ideal of $M^{*}$, we know $(x+y, z+m) \in A$ and $(x \alpha y, m \alpha v) \in A$, thus, $\mu(x+y, m+v)=0$, and $\mu(x \alpha y, m \alpha v)=0$. Since $\max \{\mu(x, m), \mu(y, v)\}=1$, and, $\min \{\mu(x, m), \mu(y, v)\}=0$, we have, $0=\mu(x+y, m+v)<\max \{\mu(x, m), \mu(y, v)\}=1$, and

$$
0=\mu(x \alpha y, m \alpha v)=\min \{\mu(x, m), \mu(y, v)\}=0
$$

hence the conclusion.

Case IV. $(x, m) \notin A$ and $(y, v) \in A$

In this case we know $\mu(x, m)=1$ and $\mu(y, v)=0$. Also since $A$ is an ideal of $M^{*}$, we know $(x+y, z+m) \in A$ and $(x \alpha y, m \alpha v) \in A$, thus, $\mu(x+y, m+v)=0$, and $\mu(x \alpha y, m \alpha v)=0$. Since $\max \{\mu(x, m), \mu(y, v)\}=1$, and, $\min \{\mu(x, m), \mu(y, v)\}=0$, we have, $0=\mu(x+y, m+v)<\max \{\mu(x, m), \mu(y, v)\}=1$, and

$$
0=\mu(x \alpha y, m \alpha v)=\min \{\mu(x, m), \mu(y, v)\}=0
$$

hence the conclusion.

Definition 2.7. Let $M$ be a $\Gamma$-semiring, and $\mu$ be an anti-fuzzy ideal of $M \times M$. We say $\mu$ is an anti-fuzzy prime ideal of $M \times M$, if $\mu(x, m) \leq \mu(x \alpha x, m \alpha m)$ for all $(x, m) \in M \times M$, and $\alpha \in \Gamma$.

Theorem 2.8. Let $M$ be a $\Gamma$-semiring, and $\mu$ be an anti-fuzzy ideal of $M \times M$. Then the following are equivalent:

(a) $\mu$ is an anti fuzzy semi prime ideal of $M \times M$.

(b) $\mu(x, m)=\mu(x \alpha x, m \alpha m)$ for all $(x, m) \in M \times M$, and $\alpha \in \Gamma$.

Proof. ((b) $\Rightarrow$ (a)) If (b) holds, then we know two inequalities are satisfied, of which one of them is, $\mu(x, m) \leq \mu(x \alpha x, m \alpha m)$ for all $(x, m) \in M \times M$, and $\alpha \in \Gamma$, hence, $\mu$ is an anti fuzzy semi prime ideal of $M \times M$.

$((a) \Rightarrow(b))$ If (a) holds, then we know the following inequality holds for all $(x, m) \in M \times M$ and $\alpha \in \Gamma$,

$$
\mu(x, m) \leq \mu(x \alpha x, m \alpha m) .
$$


Since $\mu$ is an anti fuzzy ideal of $M \times M$, we may assume $\mu$ is an anti fuzzy right ideal of $M \times M$, then we know the following inequality holds for all $(x, m),(x, m) \in M \times M$ and $\alpha \in \Gamma$,

$$
\mu(x \alpha x, m \alpha m) \leq \mu(x, m) .
$$

Thus combining the two inequalities above gives the conclusion.

Definition 2.9. Let $M$ be a $\Gamma$-semiring, and $\mu$ be a fuzzy subset of $M \times M$. Then the fuzzy subset $\langle(x, m), \mu\rangle: M \times M \mapsto[0,1]$ defined by

$$
\langle(x, m), \mu\rangle(y, v)=\sup _{\alpha \in \Gamma} \mu(x \alpha y, m \alpha v)
$$

for all $(y, v) \in M \times M$ is called an extension of $\mu$ by $(x, m)$.

Theorem 2.10. Let $M$ be a $\Gamma$-semiring, and $\mu$ be an anti fuzzy right ideal of $M \times M$. Then $\langle(x, m), \mu\rangle$ is an anti fuzzy right ideal of $M \times M$.

Proof. Let $(z, k),(y, v) \in M \times M$, and $\alpha \in \Gamma$. Now observe we have the following:

$$
\begin{aligned}
\langle(x, m), \mu\rangle(y+z, v+k) & =\sup _{\alpha \in \Gamma} \mu(x \alpha(y+z), m \alpha(v+k)) \\
& =\sup _{\alpha \in \Gamma} \mu(x \alpha y+x \alpha z, m \alpha v, m \alpha k) \\
& \leq \sup _{\alpha \in \Gamma} \max \{\mu(x \alpha y, m \alpha v), \mu(x \alpha z, m \alpha k)\} \\
& =\max _{\alpha \in \Gamma}\left\{\sup _{\alpha \in \Gamma} \mu(x \alpha y, m \alpha v), \sup _{\alpha \in \Gamma} \mu(x \alpha z, m \alpha k)\right\} \\
& \left.=\max _{\alpha \in \Gamma}\{(x, m), \mu\rangle(y, v),\langle(x, m), \mu\rangle(z, k)\right\}
\end{aligned}
$$

Also

$$
\begin{aligned}
\langle(x, m), \mu\rangle(y \alpha z, v \alpha k) & =\sup _{\beta \in \Gamma} \mu(x \beta(y \alpha z), m \beta(v \alpha k)) \\
& =\sup _{\beta \in \Gamma} \mu((x \beta y) \alpha z,(m \beta v) \alpha k) \\
& \leq \sup _{\beta \in \Gamma} \mu(x \beta y, m \beta v)
\end{aligned}
$$




$$
=\langle(x, m), \mu\rangle(y, v) .
$$

Definition 2.11. Let $M$ be a $\Gamma$-semiring, and $\mu$ be a fuzzy subset of $M \times M$. We say $\mu$ is an anti fuzzy prime ideal of $M \times M$ if

$$
\mu(x \alpha y, m \alpha v)=\min \{\mu(x, m), \mu(y, v)\}
$$

for all $(x, m),(y, v) \in M \times M$, and $\alpha \in \Gamma$.

Theorem 2.12. Let $M$ be a $\Gamma$-semiring, and $\mu$ be an anti fuzzy prime ideal of $M \times M$, and $(x, m) \in M \times M$. Then $\langle(x, m), \mu\rangle$ is an anti fuzzy prime ideal of $M \times M$.

Proof. Let $(x, m),(y, v),(z, k) \in M \times M$, and $\beta \in \Gamma$. Now observe we have the following:

$$
\begin{aligned}
\langle(x, m), \mu\rangle(y \beta z, v \beta k) & =\sup _{\alpha \in \Gamma} \mu(x \alpha(y \beta z), m \alpha(v \beta k)) \\
& =\sup _{\alpha \in \Gamma} \min \{\mu(x, m), \mu(y \beta z, v \beta k)\} \\
& =\sup _{\alpha \in \Gamma} \min \{\mu(x, m), \min \{\mu(y, v), \mu(z, k)\}\} \\
& =\sup _{\alpha \in \Gamma} \min \{\min \{\mu(x, m), \mu(y, v)\}, \min \{\mu(x, m), \mu(z, k)\}\} \\
& =\sup _{\alpha \in \Gamma} \min \{\mu(x \alpha y, m \alpha v), \mu(x \alpha z, m \alpha k)\} \\
& =\min _{\alpha \in \Gamma}\left\{\sup \mu(x \alpha y, m \alpha v), \sup _{\alpha \in \Gamma} \mu(x \alpha z, m \alpha k)\right\} \\
& =\min \{\langle(x, m), \mu\rangle(y, v),\langle(x, m), \mu\rangle(z, k)\} .
\end{aligned}
$$

Hence the theorem.

Theorem 2.13. Let $M$ be a commutative $\Gamma$-semiring, and $\mu$ be a fuzzy subset of $M \times M$. Suppose $(x, m) \in M \times M$ such that the extension $\langle(x, m), \mu\rangle=\mu$ for every $(x, m) \in M \times M$. Then $\mu$ is a constant function.

Proof. Let $M$ be a commutative $\Gamma$-semiring, and $\mu$ be a fuzzy subset of $M \times M$. Suppose $(x, m),(y, v) \in M \times M$, and $\alpha \in \Gamma$. Now observe we have the following: 


$$
\begin{aligned}
\mu(x, m) & =\langle(y, v), \mu\rangle(x, m) \\
& =\sup _{\alpha \in \Gamma} \mu(y \alpha x, v \alpha m) \\
& =\sup _{\alpha \in \Gamma} \mu(x \alpha y, m \alpha v) \\
& =\langle(x, m), \mu\rangle(y, v) \\
& =\mu(y, v) .
\end{aligned}
$$

Hence, $\mu(x, m)=\mu(y, v)$, and the theorem follows.

Definition 2.14. Let $M$ be a $\Gamma$-semiring, and $\mu$ be an anti fuzzy ideal of $M \times M$. For any $t \in[0,1]$, we define $\mu_{t}$ by the set

$$
\{(x, m) \in M \times M \mid \mu(x, m) \leq t\}
$$

and call $\mu_{t}$ an anti level subset.

Definition 2.15. Let $M$ be a $\Gamma$-semiring, $A \subseteq M \times M$, and $(x, m) \in M \times M$. We define $\langle(x, m), A\rangle$ by

$$
\{(y, v) \in M \times M \mid(x \alpha y, m \alpha v) \in A, \text { for all } \alpha \in \Gamma\} .
$$

Theorem 2.16. Let $M$ be a commutative $\Gamma$-semiring, and $\mu$ be a fuzzy subset of $M \times M$. Then for every $t \in \operatorname{Im}(\mu),\left\langle(x, m), \mu_{t}\right\rangle=\langle(x, m), \mu\rangle_{t}$ for every $(x, m) \in$ $M \times M$.

Proof. Observe we have the following

$$
\begin{aligned}
& (y, v) \in\langle(x, m), \mu\rangle_{t} \\
& \Leftrightarrow \\
& \langle(x, m), \mu\rangle(y, v) \leq t \\
& \Leftrightarrow \\
& \sup _{\alpha \in \Gamma} \mu(x \alpha y, m \alpha v) \\
& \Leftrightarrow
\end{aligned}
$$




$$
\begin{aligned}
& \mu(x \alpha y, m \alpha v) \leq t \\
& \Leftrightarrow \\
& (x \alpha y, m \alpha v) \in \mu_{t} \\
& \Leftrightarrow \\
& (y, v) \in\left\langle(x, m), \mu_{t}\right\rangle \text { by Definition } 2.15 \text {. }
\end{aligned}
$$

Hence the theorem.

Note that Theorem 2.10 holds, if $\mu$ is an anti fuzzy left ideal of $M \times M$, thus the following is immediate

Corollary 2.17. Let $M$ be a commutative $\Gamma$-semiring and $\mu$ be an anti fuzzy ideal of $M \times M$, and $(x, m) \in M \times M$. Then the extension

$$
\langle(x, m), \mu\rangle
$$

is an anti fuzzy ideal of $M \times M$.

Definition 2.18. Let $M$ be a $\Gamma$-semiring, and $\mu$ be an anti-fuzzy ideal of $M \times M$. We say $\mu$ is an anti fuzzy semi prime ideal if

$$
\mu(x, m) \leq \mu(x \alpha x, m \alpha m)
$$

for all $(x, m) \in M \times M$.

Theorem 2.19. Let $M$ be a commutative $\Gamma$-semiring, $\mu$ be an anti-fuzzy semi prime ideal of $M \times M$, and $(x, m) \in M \times M$. Then $\langle(x, m), \mu\rangle$ is an anti-fuzzy semi prime ideal of $M \times M$.

Proof. Let $M$ be a commutative $\Gamma$-semiring, $\mu$ be an anti-fuzzy semi prime ideal of $M \times M, \quad(x, m),(y, v) \in M \times M \quad$ and $\beta \in \Gamma$. By Corollary 2.17, the extension $\langle(x, m), \mu\rangle$ is an anti-fuzzy ideal of $M \times M$. Now observe we have the following:

$$
\begin{aligned}
\langle(x, m), \mu\rangle(y, v) & =\sup _{\alpha \in \Gamma} \mu(x \alpha y, m \alpha v) \\
& \leq \sup _{\alpha \in \Gamma} \mu(x \alpha y \beta x \alpha y, m \alpha v \beta m \alpha v) \\
& =\sup _{\alpha \in \Gamma} \mu(x \alpha y \beta y \alpha x, m \alpha v \beta v \alpha m)
\end{aligned}
$$




$$
\begin{aligned}
& \leq \sup _{\alpha \in \Gamma} \mu(x \alpha y \beta y, m \alpha v \beta v) \\
& =\langle(x, m), \mu\rangle(y \beta y, v \beta v) .
\end{aligned}
$$

Hence the theorem.

Definition 2.20. Let $M$ be a $\Gamma$-semiring. We say the fuzzy ideal $\mu$ of $M \times M$ is an anti fuzzy $k$-ideal of $M \times M$ if

$$
\mu(x, m) \leq \max \{\mu(x+y, m+v), \mu(y, v)\}
$$

for all $(x, m),(y, v) \in M \times M$.

Theorem 2.21. Let $M$ be a commutative $\Gamma$-semiring, $\mu$ be an anti fuzzy $k$-ideal of $M \times M$, and $(z, k) \in M \times M$. Then $\langle(z, k), \mu\rangle$ is an anti fuzzy $k$-ideal of $M \times M$.

Proof. Let $M$ be a commutative $\Gamma$-semiring, $\mu$ be an anti fuzzy $k$-ideal of $M \times M$, and $(x, m),(y, v),(z, k) \in M \times M$, and $\alpha \in \Gamma$. Based on the assumption, it follows from Corollary 2.17 that the extension $\langle(z, k), \mu\rangle$ is an anti fuzzy ideal of $M \times M$. Now we know the following for all $(x, m),(y, v) \in M \times M$

$$
\mu(x, m) \leq \max \{\mu(x+y, m+v), \mu(y, v)\} .
$$

Thus for all $(x, m),(y, v) \in M \times M$, and $\alpha \in \Gamma$, we deduce the following:

$$
\mu(z \alpha x, k \alpha m) \leq \max \{\mu(z \alpha x+z \alpha y, k \alpha m+k \alpha v), \mu(z \alpha y+k \alpha v)\} .
$$

Now taking sup on both sides of the above inequality over all $\alpha \in \Gamma$, we deduce the following:

$$
\sup _{\alpha \in \Gamma} \mu(z \alpha x, k \alpha m) \leq \max \left\{\sup _{\alpha \in \Gamma} \mu(z \alpha x+z \alpha y, k \alpha m+k \alpha v), \sup _{\alpha \in \Gamma} \mu(z \alpha y+k \alpha v)\right\} .
$$

By Definition 2.9, the above inequality translates as

$$
\langle(z, k), \mu\rangle(x, m) \leq \max \{\langle(z, k), \mu\rangle(x+y, m+v),\langle(z, k), \mu\rangle(y, v)\} .
$$

Hence the theorem.

Theorem 2.22. Let $M$ be a commutative $\Gamma$-semiring, and $\mu$ be an anti fuzzy ideal of $M \times M$. If for $(y, v) \in M \times M, \mu(y, v)$ is not minimal in $\mu(M \times M)$, and $\langle(x, m), \mu\rangle$ $=\mu$, then $\mu$ is an anti-fuzzy prime ideal of $M \times M$. 
Proof. Let $\left(a, a^{\prime}\right),\left(b, b^{\prime}\right) \in M \times M$, and $\alpha \in \Gamma$. We know

$$
\sup _{\alpha \in \Gamma} \mu\left(a \alpha b, a^{\prime} \alpha b^{\prime}\right)=\left\langle\left(a, a^{\prime}\right), \mu\right\rangle\left(b, b^{\prime}\right)=\mu\left(a, a^{\prime}\right)
$$

and

$$
\sup _{\alpha \in \Gamma} \mu\left(b \alpha a, b^{\prime} \alpha a^{\prime}\right)=\left\langle\left(b, b^{\prime}\right), \mu\right\rangle\left(a, a^{\prime}\right)=\mu\left(b, b^{\prime}\right) .
$$

We also know $\mu\left(a \alpha b, a^{\prime} \alpha b^{\prime}\right) \leq \mu\left(a, a^{\prime}\right)$ and $\mu\left(a \alpha b, a^{\prime} \alpha b^{\prime}\right) \leq \mu\left(b, b^{\prime}\right)$.

Case I. $\mu\left(a, a^{\prime}\right)$ or $\mu\left(b, b^{\prime}\right)$ is not minimal in $\mu(M \times M)$

If $\mu\left(a, a^{\prime}\right)$ is not minimal, then since

$$
\sup _{\alpha \in \Gamma} \mu\left(b \alpha a, b^{\prime} \alpha a^{\prime}\right)=\left\langle\left(b, b^{\prime}\right), \mu\right\rangle\left(a, a^{\prime}\right)=\mu\left(b, b^{\prime}\right)
$$

and $M$ is a commutative $\Gamma$-semiring, then

$$
\begin{aligned}
\mu\left(a \alpha b, a^{\prime} \alpha b^{\prime}\right) & =\mu\left(b \alpha a, b^{\prime} \alpha a^{\prime}\right) \geq \sup _{\alpha \in \Gamma} \mu\left(b \alpha a, b^{\prime} \alpha a^{\prime}\right) \\
& =\left\langle\left(b, b^{\prime}\right), \mu\right\rangle\left(a, a^{\prime}\right)=\mu\left(b, b^{\prime}\right)
\end{aligned}
$$

and since we know $\mu\left(a \alpha b, a^{\prime} \alpha b^{\prime}\right) \leq \mu\left(b, b^{\prime}\right)$, hence,

$$
\mu\left(a \alpha b, a^{\prime} \alpha b^{\prime}\right)=\mu\left(b, b^{\prime}\right)=\min \left\{\mu\left(b, b^{\prime}\right), \mu\left(a, a^{\prime}\right)\right\} .
$$

If $\mu\left(b, b^{\prime}\right)$ is not minimal, then in a similar way we can conclude

$$
\mu\left(a \alpha b, a^{\prime} \alpha b^{\prime}\right)=\mu\left(a, a^{\prime}\right)=\min \left\{\mu\left(b, b^{\prime}\right), \mu\left(a, a^{\prime}\right)\right\} .
$$

Hence the theorem.

Case II. Neither $\mu\left(a, a^{\prime}\right)$ nor $\mu\left(b, b^{\prime}\right)$ is minimal in $\mu(M \times M)$

Since we know $\mu\left(a \alpha b, a^{\prime} \alpha b^{\prime}\right) \leq \mu\left(a, a^{\prime}\right)$ and $\mu\left(a \alpha b, a^{\prime} \alpha b^{\prime}\right) \leq \mu\left(b, b^{\prime}\right)$, it follows that

$$
\mu\left(a \alpha b, a^{\prime} \alpha b^{\prime}\right) \leq \min \left\{\mu\left(a, a^{\prime}\right), \mu\left(b, b^{\prime}\right)\right\}
$$

Now from

$$
\sup _{\alpha \in \Gamma} \mu\left(a \alpha b, a^{\prime} \alpha b^{\prime}\right)=\sup _{\alpha \in \Gamma} \mu\left(b \alpha a, b^{\prime} \alpha a^{\prime}\right)=\left\langle\left(b, b^{\prime}\right), \mu\right\rangle\left(a, a^{\prime}\right)=\mu\left(b, b^{\prime}\right)
$$


and

$$
\sup _{\alpha \in \Gamma} \mu\left(a \alpha b, a^{\prime} \alpha b^{\prime}\right)=\left\langle\left(a, a^{\prime}\right), \mu\right\rangle\left(b, b^{\prime}\right)=\mu\left(a, a^{\prime}\right)
$$

we deduce that

$$
\mu\left(a \alpha b, a^{\prime} \alpha b^{\prime}\right) \geq \mu\left(a, a^{\prime}\right) \text { and } \mu\left(a \alpha b, a^{\prime} \alpha b^{\prime}\right) \geq \mu\left(b, b^{\prime}\right)
$$

hence

$$
\mu\left(a \alpha b, a^{\prime} \alpha b^{\prime}\right) \geq \min \left\{\mu\left(a, a^{\prime}\right), \mu\left(b, b^{\prime}\right)\right\} .
$$

Consequently, we have

$$
\mu\left(a \alpha b, a^{\prime} \alpha b^{\prime}\right)=\min \left\{\mu\left(b, b^{\prime}\right), \mu\left(a, a^{\prime}\right)\right\}
$$

hence the theorem

\section{Concluding Remark and Further Direction}

Given $M$ is a $\Gamma$-semiring, we have introduced concepts of anti-fuzzy prime ideal, anti-fuzzy semi prime ideal, and anti-fuzzy ideal extension, respectively, of $M \times M$; some properties associated with these new concepts have been obtained.

A future interesting problem is introduce some concepts of triple $\Gamma$-semiring $(M \times M \times M)$, and study some of their properties.

\section{References}

[1] Clement Boateng Ampadu, Some properties of anti fuzzy ideal and anti fuzzy $k$ ideal of couple gamma-semirings, Journal of New Theory (to appear).

[2] M. Murali Krishna Rao and B. Venkateswarlu, Anti fuzzy ideal extension of $\Gamma$-semiring, Bulletin of the International Mathematical Virtual Institute 4 (2014), 135-144. 